\title{
P20-02. Characterization of phenotypic, genotypic and neutralization sensitivity of the HIV-I CRF07_B'C Strains circulating in the Xinjiang Province of China

\author{
$\mathrm{L} \mathrm{Ma}^{* 1}$, Y Guo ${ }^{2}$, S Sun ${ }^{1}$, L Yuan ${ }^{1}$, Y Huang1, S Qu ${ }^{1}, \mathrm{X} \mathrm{Yu}{ }^{1}, \mathrm{Z}$ Meng1, X He${ }^{1}$, \\ $S$ Jiang ${ }^{3}$ and $Y$ Shao $^{1}$
}

Address: ${ }^{1}$ National Center for AIDS/STD Control and Prevention (NCAIDS), Chinese Center for Disease Control and Prevention (China-CDC), Beijing, PR China, ${ }^{2}$ the First Affiliated Hospital of Xinjiang Medical University, Urumqi, PR China and ${ }^{3}$ Lindsley F. Kimball Research Institute, New York Blood Center, New York, USA

* Corresponding author

from AIDS Vaccine 2009

Paris, France. 19-22 October 2009

Published: 22 October 2009

Retrovirology 2009, 6(Suppl 3):P372 doi:I0.II86/I742-4690-6-S3-P372

This abstract is available from: http://www.retrovirology.com/content/6/S3/P372

(c) 2009 Ma et al; licensee BioMed Central Ltd.

\section{Background}

HIV-1 CRF07_B'C recombinant previously circulated mainly among the intravenous drug users (IDUs) in Northwest China and is currently spreading in the entire country. The aim of this study is to characterize the genotypic, phenotypic properties as well as neutralization profile of virus strains in comparison with the subtype B' (Thailand B) which is prevalent in the former plasma donors (FPDs) in centre China.

\section{Methods}

12 CRF07_BC and 20 subtype B' HIV-1 strains were isolated from Xinjiang and Anhui. Replication kinetics of the generated virus stock was performed every 2-3 days in PHA-stimulated PBMCs. HIV-1 Co-receptor usage was detected using Ghost cells, and a sensitive HIV-1 neutralization assay based on TZM-b1 cells was performed to detect the neutralization activity of plasma from patients infected with autologous and heterologous viruses.

\section{Results}

12 CRF07_B'C viruses utilized CCR5 co-receptor, while they had lower net charge value in the V3 loop and exhibited slower replication kinetics than subtype B' viruses. The number and location of the potential N-linked glycosylation sites in $\mathrm{V} 1 / \mathrm{V} 2$ and the $\mathrm{C} 2$ region of the CRF07_B'C viruses were significantly different from those of the subtype $\mathrm{B}^{\prime}$ viruses. The plasmas from patients infected with CRF07_B'C virus effectively neutralized more than fifty percent of heterologous viruses but failed to neutralize autologous HIV-1. The neutralizing activity of their plasmas was negatively corrected with neutralization sensitivity of HIV-1 isolates from the patents. Ten percent of the plasmas with potent neutralizing activity against autologous viurses were also effective in neutralizing infection by HIV-1 subtypes besides B' and B'/C.

\section{Conclusion}

This study suggests that CRF07_B'C may be superior over $\mathrm{B}^{\prime}$ in stimulating cross clade neutralizing antibody. It also indicates that some of these HIV-1 viruses can elicit both intragroup and cross-clade neutralizing antibodies. 